\title{
Sub and Super-Luminal Propagation of Intense Pulses in Media with Saturated and Reverse Absorption
}

\author{
G.S.Agarwal, and Tarak Nath Dey \\ Physical Research Laboratory, Navrangpura, Ahmedabad-380 009, India
}

(August 16, 2018)

\begin{abstract}
We develop models for the propagation of intense pulses in solid state media which can have either saturated absorption or exhibit reverse absorption. We show that the experiments of Bigelow et al.[Phys. Rev. Lett. 90, 113903 (2003); Science 301, 200 (2003).] on subluminal propagation in Ruby, and superluminal propagation in Alexandrite are well explained by modelling them as three level and four level systems respectively, coupled to Maxwell equations. We present results well beyond the traditional pump-probe approach.
\end{abstract}

Since the discovery of ultraslow light with a group velocity $17 \mathrm{~m} / \mathrm{sec}$ in a Bose condensate by Hau et al. [1-3] many experiments have reported slow light in a variety of media [4-6]. Kash et al. [4] demonstrated light propagation with a group velocity of $90 \mathrm{~m} / \mathrm{sec}$ at room temperature in Rb vapor. Using Zeeman coherences, Budker et al. [5] reported slow light with group velocity $8 \mathrm{~m} / \mathrm{sec}$ in Rb vapor. Hemmer et al. [6] first reported slow light in solid state material namely $\mathrm{Pr}$ doped $\mathrm{Y}_{2} \mathrm{SiO}_{5}$, maintained at a cryogenic temperature of $5 \mathrm{~K}$. The slow light ideas have been successfully used in storage and retrieval of light pulses $[7,8]$. The understanding of storage and retrieval of light pulses has been provided by Dey and Agarwal [9], using the adiabaton theory of Grobe, Hioe and Eberly [10].

Work on pulse propagation continues to produce interesting results [11-14]. Recently, Bigelow et al. [15] showed the propagation of light pulses in Ruby at a group velocity of $57.5 \mathrm{~m} / \mathrm{sec}$. This experiment differs considerably from all earlier experiments which were based on electromagnetically induced transparency [16]. Bigelow et al. recognize that a two level system driven by a strong field and a probe gives rise to a hole in the probe response function with a width of the order of $1 / T_{1}$, where $T_{1}$ is the longitudinal relaxation time [17]. Note that in a material like Ruby the transverse relaxation time $T_{2} \ll T_{1}$. These authors also discovered that they need not use separate pump and probe fields. A field with peak power of the order of saturation intensity could be slowed down considerably to about $57.5 \mathrm{~m} / \mathrm{sec}$. Further Bigelow et al. [18] extended their work to a material like Alexandrite, where they reported superluminal propagation. There have been several earlier reports of superluminal propagation in solid state systems [19]; and in vapors [20,21].

The purpose of this letter is to study the propagation of intense pulses in a homogeneously broadened medium, like Ruby, which can exhibit saturated absorption or a medium like Alexandrite, which can exhibit reverse absorption. Note that the pulse propagation in a nonlinear transparent medium has been extensively studied [22]. The systems studied here differ from the previous studies as our systems posses very strong transverse and longitudinal relaxation effects. In order to model the experiments, we model Ruby as a three level system and Alexandrite as a four level system. We solve the system of coupled equations numerically to delineate various aspects of pulse propagation. We do not make any approximation on the strength of the pulses so that we can model experimental observations on strong pulses. We calculate group velocity from the relative delay or advancement between the reference pulse and the output pulse. We present numerical results on the propagation of Gaussian and modulated pulses and show good agreement with the experimental data of Refs. $[15,18]$. The experiments of Bigelow et al. fall in two categories. One consists of a weak probe and a strong coherent $\mathrm{cw}$ field. These were explained in terms of the response to a probe field of a two level medium pumped by a coherent field [23]. The other category consists of the self delay of a strong pulse. The latter requires solutions of the coupled Maxwell-Bloch equations and this is the case we concentrate on.

Media with saturated absorption - -For pulse propagation in the three level model in Ruby, we represent the ground state as $|g\rangle$, the ${ }^{4} F_{2}$ absorption band as $\left|e_{1}\right\rangle$ and the levels $2 \bar{A}$ and $\bar{E}$ as $\left|e_{2}\right\rangle$. In Ruby one has very rapid decay of the level $\left|e_{1}\right\rangle$ to $\left|e_{2}\right\rangle$, and as a result some of the coherences become irrelevant on experimental time scale. The density matrix equations for the model of the Fig. 1 are

$$
\begin{aligned}
& \dot{\rho}_{g g}=2 \Gamma_{2} \rho_{22}+i \Omega\left(\rho_{1 g}-\rho_{g 1}\right) \\
& \dot{\rho}_{22}=2 \Gamma_{1} \rho_{11}-2 \Gamma_{2} \rho_{22} \\
& \dot{\rho}_{1 g}=-\Gamma_{1} \rho_{1 g}+i \Omega\left(\rho_{g g}-\rho_{11}\right) \\
& \rho_{g g}+\rho_{11}+\rho_{22}=1,
\end{aligned}
$$

where $\rho_{i j}=\left\langle e_{i}|\rho| e_{j}\right\rangle, i, j=1,2$. The Rabi frequency $2 \Omega$ is defined by $2 \Omega(z, t)=2 \vec{d}_{1 g} \cdot \overrightarrow{\mathcal{E}}(z, t) / \hbar$, where $\vec{d}_{1 g}$ is the dipole matrix element and $\overrightarrow{\mathcal{E}}(z, t)$ is the envelop of the pulse. We assume that the carrier frequency, $\omega$, is on resonance 
with the frequency of the $\left|e_{1}\right\rangle \longleftrightarrow|g\rangle$ transition. Under the approximations, $\Gamma_{1} \gg \Gamma_{2}, \Omega ; \dot{\rho}_{1 g} \sim 0$, we derive the approximate equation for the evolution of the ground state population as

$$
\dot{\rho}_{g g}=2 \Gamma_{2}\left(1-\rho_{g g}\right)-2 \frac{\Omega^{2}}{\Gamma_{1}} \rho_{g g}
$$

Note that we can prove that $\dot{\rho}_{11} \approx 0$, if $\Gamma_{1} \gg \Gamma_{2}, \Omega$. Under the same conditions and the slowly varying envelop approximation, the evolution equation for the Rabi frequency of the field is governed by

$$
\frac{\partial \tilde{\Omega}}{\partial z}=-\frac{\alpha_{0}}{2} \tilde{\Omega} \rho_{g g}, \quad \tilde{\Omega}=\Omega / \Omega_{s a t}
$$

where $\alpha_{0}=4 \pi \omega\left|d_{1 g}\right|^{2} / c \hbar \Gamma_{1}$ and $\Omega_{\text {sat }}=2 \sqrt{\Gamma_{1} \Gamma_{2}}$. In Eqs. (2) and (3) we have used the pulse coordinates i.e, $t-z / c, z$. The time derivative in Eq. (2) is with respect to $(t-z / c)$. The time $t$ can be expressed in units of $1 / 2 \Gamma_{2}$. For numerical computation, we consider two types of input pulses, viz, a Gaussian pulse with a temporal width $\gtrsim 1 / \Gamma_{2}$

$$
\tilde{\Omega}_{i n}=\tilde{\Omega}^{0} e^{\left[-t^{2} / 2 \sigma^{2}\right]}
$$

and amplitude modulated pulse

$$
\tilde{\Omega}_{i n}^{2}(t)=I=I_{0}(1+m \cos [\Delta t]) .
$$

The Equations (2) - (5) are our working equations. We use these for numerical computations. We calculate the evolution of the pulse for arbitrary values of $\tilde{\Omega}^{0}$ or $I_{0}$. Some typical results for the Gaussian pulses are shown in the Fig. 2. We get group velocities in the range $50 \mathrm{~m} / \mathrm{sec}$ for $\Omega / \Omega_{\text {sat }} \sim 1$ and the transmission is rather small. In Fig. 3 , we exhibit the behavior of $v_{g}$ and transmission as a function of the input intensity. These results are in agreement with the experimental findings of transmission in the range $0.1 \%$. In Fig. 3, we also show for comparison the results of the group velocity and the transmission for the propagation of an intense pulse through a two level system described by the traditional Bloch equations. The coupled Maxwell-Bloch equations under the approximation $T_{1} \gg T_{2}$ are given by

$$
\begin{aligned}
\dot{\rho}_{11} T_{1} & =-\rho_{11}+2|\tilde{\Omega}|^{2}\left(1-2 \rho_{11}\right) \\
\rho_{g g} & +\rho_{11}=1 \\
\frac{\partial \tilde{\Omega}}{\partial z} & =-\frac{\alpha_{0}}{2} \tilde{\Omega}\left(1-2 \rho_{11}\right)
\end{aligned}
$$

where $\tilde{\Omega}=\Omega \sqrt{T_{1} T_{2}}$ and dot denotes $\partial / \partial(t-z / c)$. As seen from the Fig. 3, there are substantial differences in the propagation of pulses in two level and three level media. Note that the time $T_{1}$ is equal to $1 / 2 \Gamma_{2}$. We believe that, in the light of the energy level diagram of Ruby, it is more appropriate to model it as a three level system.

We next consider input pulse as a modulated pulse given in Eq. (5). The output pulse is modulated with a phase shift (time delay). We show this time delay as a function of modulation frequency for two different pump powers in the Fig. 4. The results in Figs. $2-4$ are in excellent agreement with the experimental data( $c f$ for example Fig. 3 of Ref. [15] with our Fig. 4).

Media with reverse saturation --For the superluminal propagation in Alexandrite, Bigelow et al. recognized how the reverse saturation mechanism [24] can be at work in a material like $\mathrm{BeAl}_{2} \mathrm{O}_{4}$ doped with $\mathrm{Cr}^{3+}$ ions and with some $\mathrm{Cr}^{3+}$ ions replaced by $\mathrm{Al}^{3+}$. The reverse saturation produces an antihole in the susceptibility for the probe in presence of a pump field. The antihole can result in the superluminal propagation. In what follows we show how the measurement can follow by modelling the system as a four level system to account for reverse absorption. The model is shown in the Fig. 5, where state ${ }^{4} A_{2}$ as $|g\rangle$, the absorption bands ${ }^{4} T_{2}$ and ${ }^{4} T_{1}$ as $\left|e_{1}\right\rangle$ and the level ${ }^{2} E$ as $\left|e_{2}\right\rangle$. The density matrix equations are now given by

$$
\begin{aligned}
& \dot{\rho}_{g g}=2 \Gamma_{2} \rho_{22}+i \Omega\left(\rho_{1 g}-\rho_{g 1}\right) \\
& \dot{\rho}_{22}=2 \Gamma_{1} \rho_{11}-2 \Gamma_{2} \rho_{22}+2 \Gamma_{3} \rho_{33} \\
& \dot{\rho}_{33}=-2 \Gamma_{3} \rho_{33}+i \Omega\left(\rho_{23}-\rho_{32}\right) \\
& \dot{\rho}_{32}=-\Gamma_{3} \rho_{32}+i \Omega\left(\rho_{22}-\rho_{33}\right) \\
& \dot{\rho}_{1 g}=-\Gamma_{1} \rho_{1 g}+i \Omega\left(\rho_{g g}-\rho_{11}\right) \\
& \rho_{g g}+\rho_{11}+\rho_{22}+\rho_{33}=1 .
\end{aligned}
$$


Following the same procedure as in the case of Ruby, we have derived the working equations

$$
\begin{aligned}
& \frac{\dot{\rho}_{g g}}{2 \Gamma_{2}}=\left(1-\rho_{g g}\right)-2 \tilde{\Omega}^{2} \rho_{g g} \\
& \frac{\partial \tilde{\Omega}}{\partial z}=-\frac{\alpha_{0}}{2} \tilde{\Omega} \rho_{g g}-\frac{\tilde{\alpha}_{0}}{2} \tilde{\Omega}\left(1-\rho_{g g}\right),
\end{aligned}
$$

where $\tilde{\alpha}_{0}$ gives the reverse saturation. Following the experimental data of Bigelow et al. [18], we estimate $\left(\tilde{\alpha}_{0} / \alpha_{0}\right) \approx 4$ . The Eqs. (10) and (11) are numerically integrated for the input Gaussian pulse given by Eq. (4). A representative set of results is shown in the Fig. 6. This Figure also shows how the group velocity and net transmission depends on the peak intensity of the Gaussian pulses. It should be borne in mind that in the range of the intensities of Fig. 6 , no perturbation theory can be used. One has to study the full nonlinear behavior. We also notice that the input pulses get distorted in shape. The distortion becomes more pronounced as the nonlinearity of the medium becomes more pronounced.

In conclusion, we have shown how to model the propagation of intense pulse in solid state media with very strong relaxation effects. The media can exhibit either saturated absorption or reverse absorption.Our modelling goes well beyond the traditional pump-probe approach. We specifically present results on the propagation of pulses in Ruby and Alexandrite. Our model would also be applicable to other systems where reverse absorption could be dominant.

GSA is grateful to R. Boyd for extensive discussions on experiments and the contents of this paper. GSA also thanks E. Wolf for partially supporting visit to Rochester through US Air Force Office of Scientific Research, grant no. F49620-03-1-0318 and the Engineering Research Program of the Office of Basic Energy Sciences at the US Department of Energy under grant no. DE-FG02-ER 45992.

[1] L. V. Hau, S. E. Harris, Z. Dutton, and C. H. Behroozi, Nature (London) 397, 594 (1999).

[2] A. Kasapi, M. Jain, G. Y. Yin, and S. E. Harris, Phys. Rev. Lett. 74, 2447 (1995); O. Schmidt, R. Wynands, Z. Hussein, and D. Meschede, Phys. Rev. A 53, R27 (1996).

[3] A. B. Mastko, O. Kocharovskaya, Y. Rostovtsev, A. S. Zibrov, and M. O. Scully, Adv. At., Mol., Opt. Phys. 46, 191 (2001); R. W. Boyd and G. J. Gauthier, in slow and Fast Light, Progress in Optics Vol. 43, edited by E. Wolf (Elsevier Amsterdam, 2002).

[4] M. M. Kash, V. A. Sautenkov, A. S. Zibrov, L. Hollberg, G. R. Welch, M. D. Lukin, Y. Rostovtsev, E. S. Fry, and M. O. Scully, Phys. Rev. Lett. 82, 5229 (1999).

[5] D. Budker, D. F. Kimball, S. M. Rochester, and V. V. Yashchuk, Phys. Rev. Lett. 83, 1767 (1999).

[6] A. V. Turukhin, V. S. Sudarshanam, M. S. Shahriar, J. A. Musser, B. S. Ham and P. R. Hemmer, Phys. Rev. Lett. 88, $023602(2002)$.

[7] C. Liu, Z. Dutton, C. H. Behroozi, and L. V. Hau, Nature (London) 409, 490 (2001).

[8] M. Fleischhauer and M. D. Lukin, Phys. Rev. Lett. 84, 5094 (2000); M. Fleischhauer and M. D. Lukin, Phys. Rev. A 65, 022314 (2002); D. F. Phillips, A. Fleischhauer, A. Mair, R. L. Walsworth, and M. D. Lukin, Phys. Rev. Lett. 86, 783 (2001).

[9] T. N. Dey and G. S. Agarwal, Phys. Rev. A 67, 033813 (2003).

[10] R. Grobe, F. T. Hioe, and J. H. Eberly, Phys. Rev. Lett. 73, 3183 (1994).

[11] E. Cerboneschi, F. Renzoni, and E. Arimondo, J. Opt. B 4, S267 (2002).

[12] J. H. Eberly and V. V. Kozlov, Phys. Rev. Lett. 88, 243604 (2002); A. Rahman and J. H. Eberly, Phys. Rev. A 58, R805 (1998).

[13] G. Juzeliunas, and H. J. Carmichael, Phys. Rev. A 65, 021601(R) (2002).

[14] C. Mewes and M. Fleischhauer, Phys. Rev. A 66, 033820 (2002).

[15] M. S. Bigelow, N. N. Lepeshkin, and R. W. Boyd, Phys. Rev. Lett. 90, 113903 (2003).

[16] S. E. Harris, Physics Today, 50(7), 36 (1997).

[17] L. W. Hillman, R. W. Boyd, J. Krasinski, and C. R. Stroud, Jr. , Opt. Commun. 45, 416 (1983).

[18] M. S. Bigelow, N. N. Lepeshkin, and R. W. Boyd, Science, 301, 200 (2003).

[19] S. Chu and S. Wong, Phys. Rev. Lett. 48, 738 (1982).

[20] L. J. Wang, A. Kuzmich, and A. Dogariu, Nature (London) 406, 277 (2000); A. Dogariu, A.Kuzmich, and L. J. Wang, Phys. Rev. A 63, 053806 (2001).

[21] For theoretical work on superluminal propagation see R. Y. Chiao, Phys. Rev. A 48, R34 (1993); E. L. Bolda, R. Y. Chiao, and J. C. Garrison, Phys. Rev. A 48, 3890 (1993); G. S. Agarwal, T. N. Dey, and S. Menon, Phys. Rev. A 64, 053809 (2001); D. Bortman-Arbiv, A. D. Wilson-Gordon, and H. Friedmann, Phys. Rev. A 63, 043818 (2001). 
[22] L. Allen and J. H. Eberly, Optical Resonance and Two Level Atoms (Dover, New York, 1987), p. 90.

[23] B. R. Mollow, Phys. Rev. A 5, 2217 (1972).

[24] M. S. Malcuit, R. W. Boyd, L. W. Hillman, and C. R. Stroud, Jr., JOSA B 1 73, (1984); K. V. Yumashev, N. V. Kuleshov, P. V. Prokoshin, A. M. Malyarevich, and V. P. Mikhailov, Appl. Phys. Lett. 70, 2523 (1997); Z. Burshtein, P. Blau, Y. Kalisky, Y. Shimony, and M. R. Kokta, IEEE J. Quantum Electron, 34, 292 (1998).

FIG. 1. Three level model for Ruby Crystal

FIG. 2. The solid curve shows light pulse propagating at speed c through $7.25 \mathrm{~cm}$ in vacuum. The long dashed and dot-dashed curves show light pulses propagating through a medium of length $7.25 \mathrm{~cm}$ at different input amplitudes. The temporal width $\sigma$ of the Gaussian pulse is $20 \mathrm{msec}$ and $1 / 2 \Gamma_{2}=4.45 \mathrm{msec}$. The part (b) gives the amplitudes, of the output pulse normalized to the input amplitudes. The transmission increases with increasing the input field intensity.

FIG. 3. Variation of transmissions and group Velocities as functions of the input amplitude of the light pulse. The solid (dashed) curve gives the intensity transmission of the pulse for the two (three) level model of the medium. The corresponding group velocities are given by the dotted curve (two level model) and the long dashed curve (three level model). The light pulse is propagating through the medium of length $\mathrm{L}=7.25 \mathrm{~cm}$.

FIG. 4. Time delay of the light pulse as a function of modulation frequency for two different input powers. The modulation index, $\mathrm{m}$, is equal to 0.05 . Note that the output pulse can be fitted to the from $I(t)=I_{o}(t)(1+m \cos (\Delta t+\theta))$. The overall transmission is small, e.g., $I_{o}(t) / I_{s}=0.00165$ for $\Delta / 2 \Gamma_{2}=2$

FIG. 5. Four level model for Alexandrite crystal

FIG. 6. The solid curve of (a) shows light pulse propagating at speed c through a distance of $7.25 \mathrm{~cm}$ in vacuum. The dotted, long dashed and dot-dashed curves depict light pulse propagating through a medium of length $7.25 \mathrm{~cm}$ at different input amplitudes. The pulse width $\sigma$ is $500 \mu \mathrm{sec}$, whereas $1 / 2 \Gamma_{2}=250 \mu \mathrm{sec}$. Fig (b) shows the amplitude of the output pulse normalized with input amplitude. The transmission is decreased on increasing the input field intensity . 


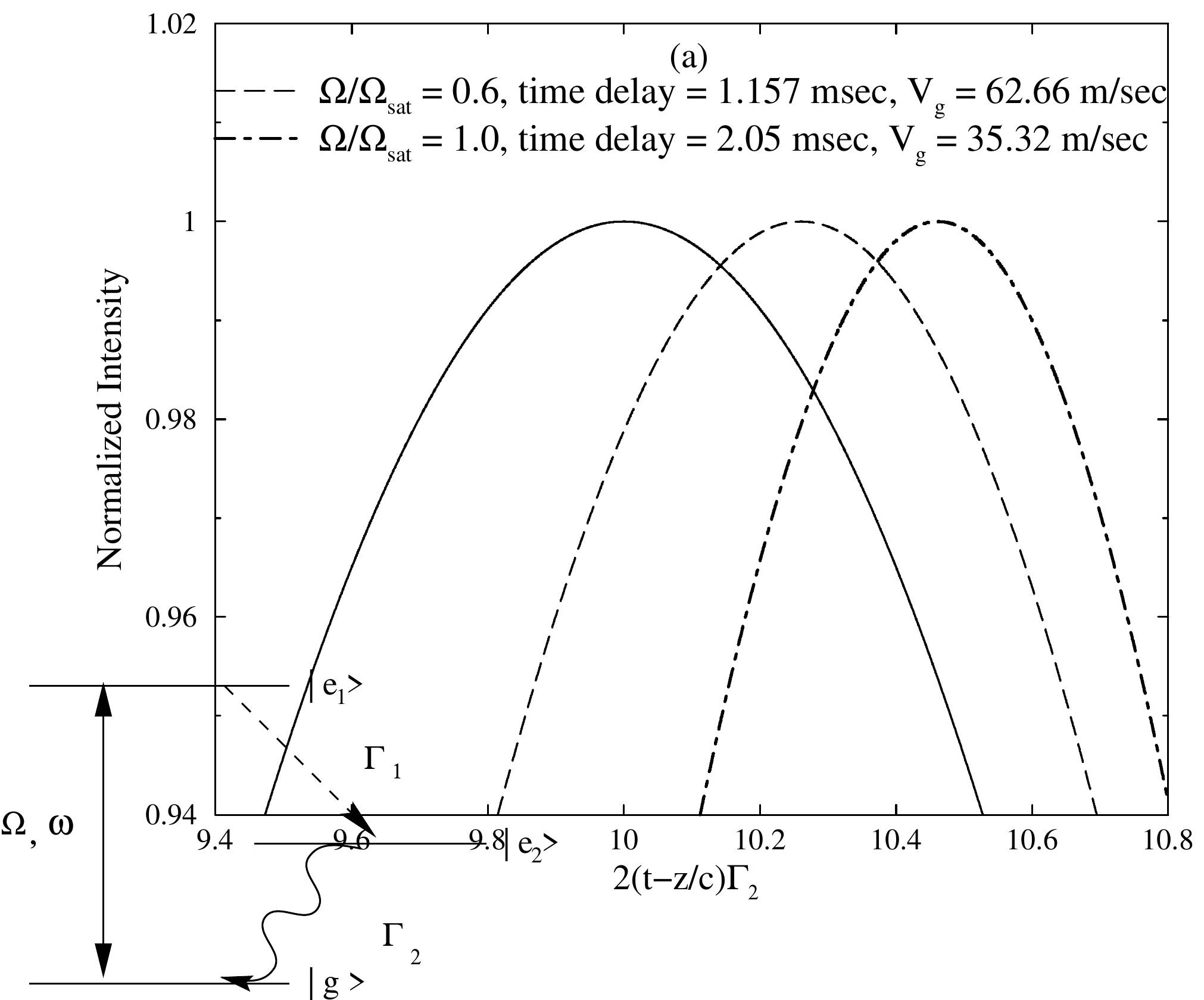




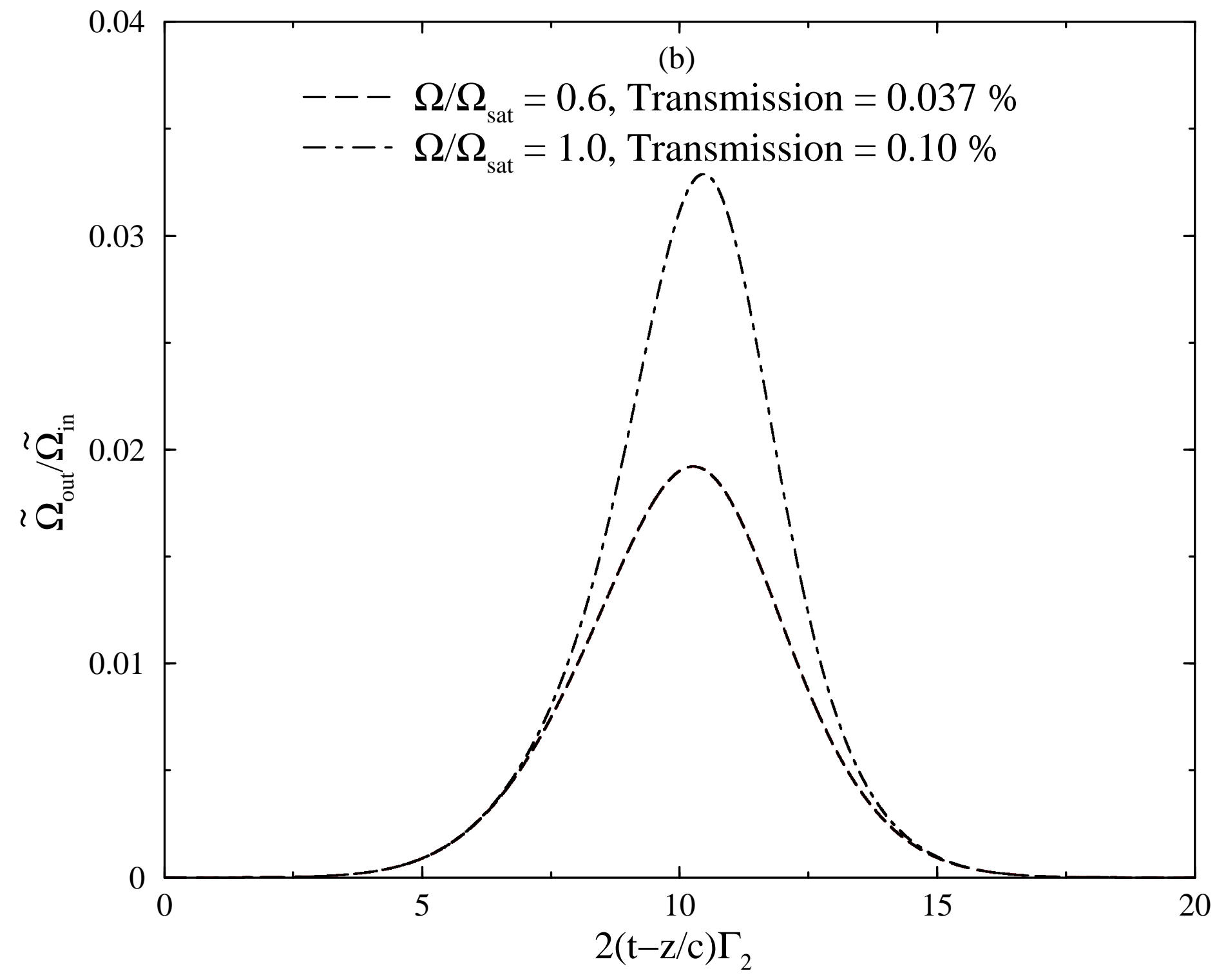




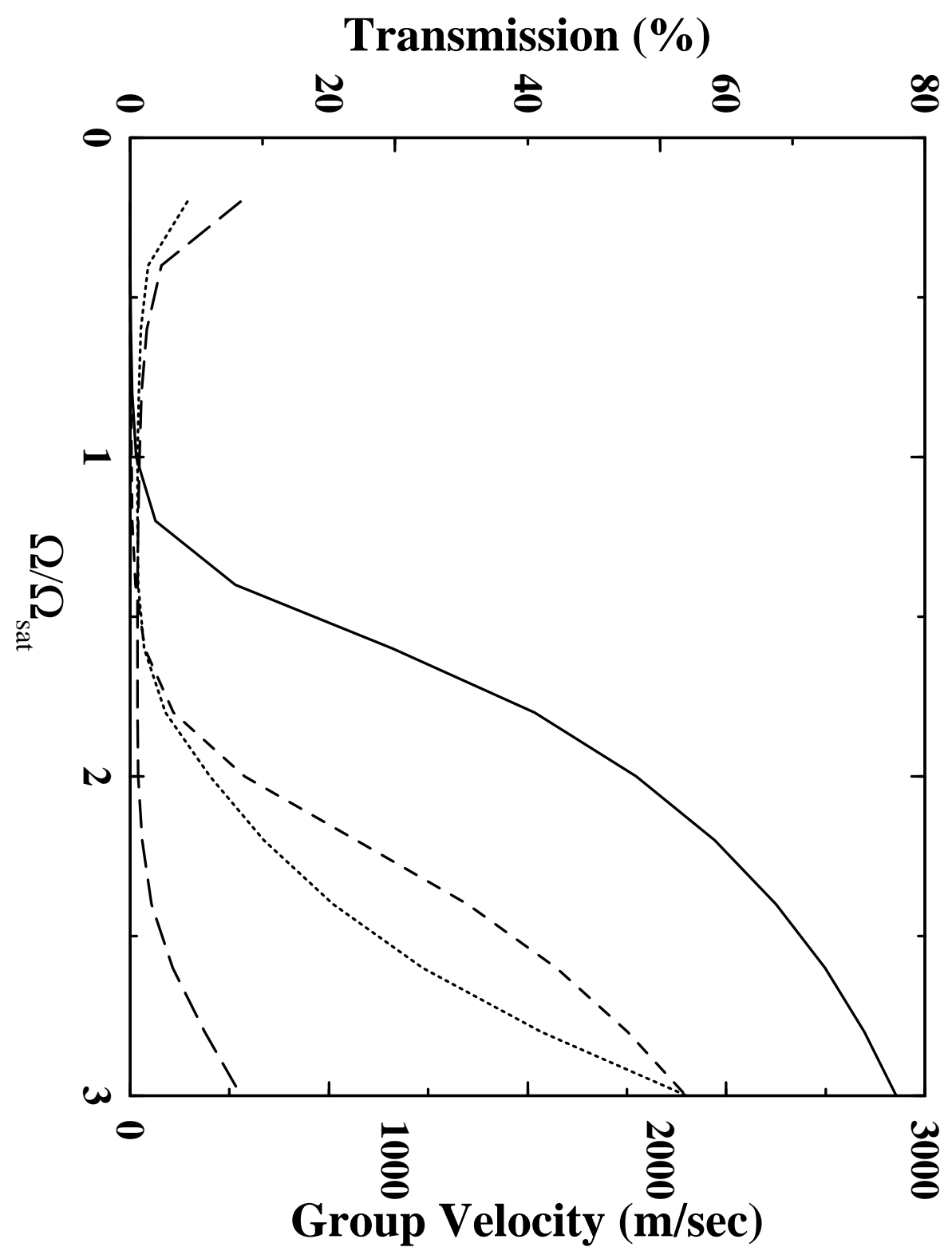




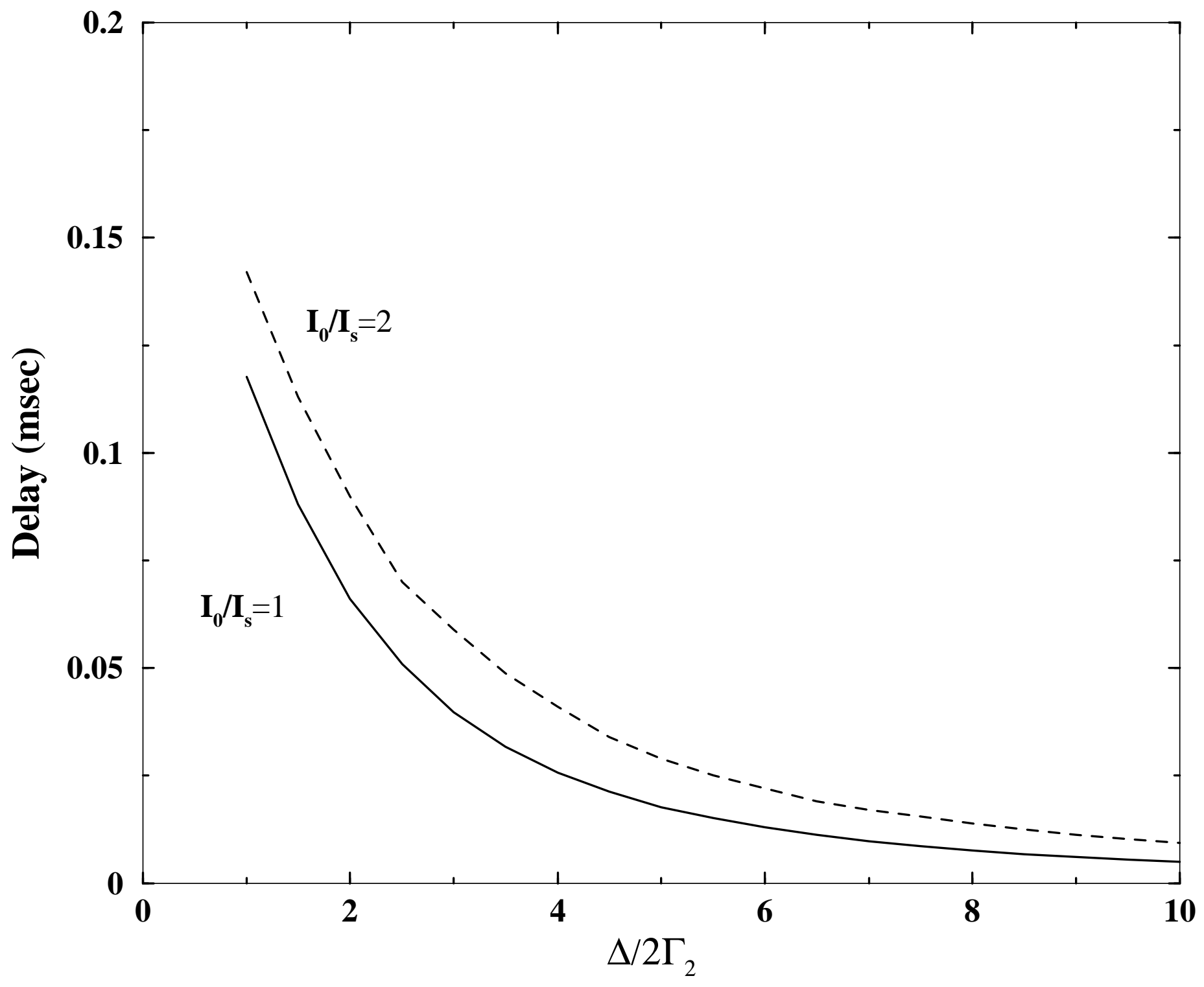




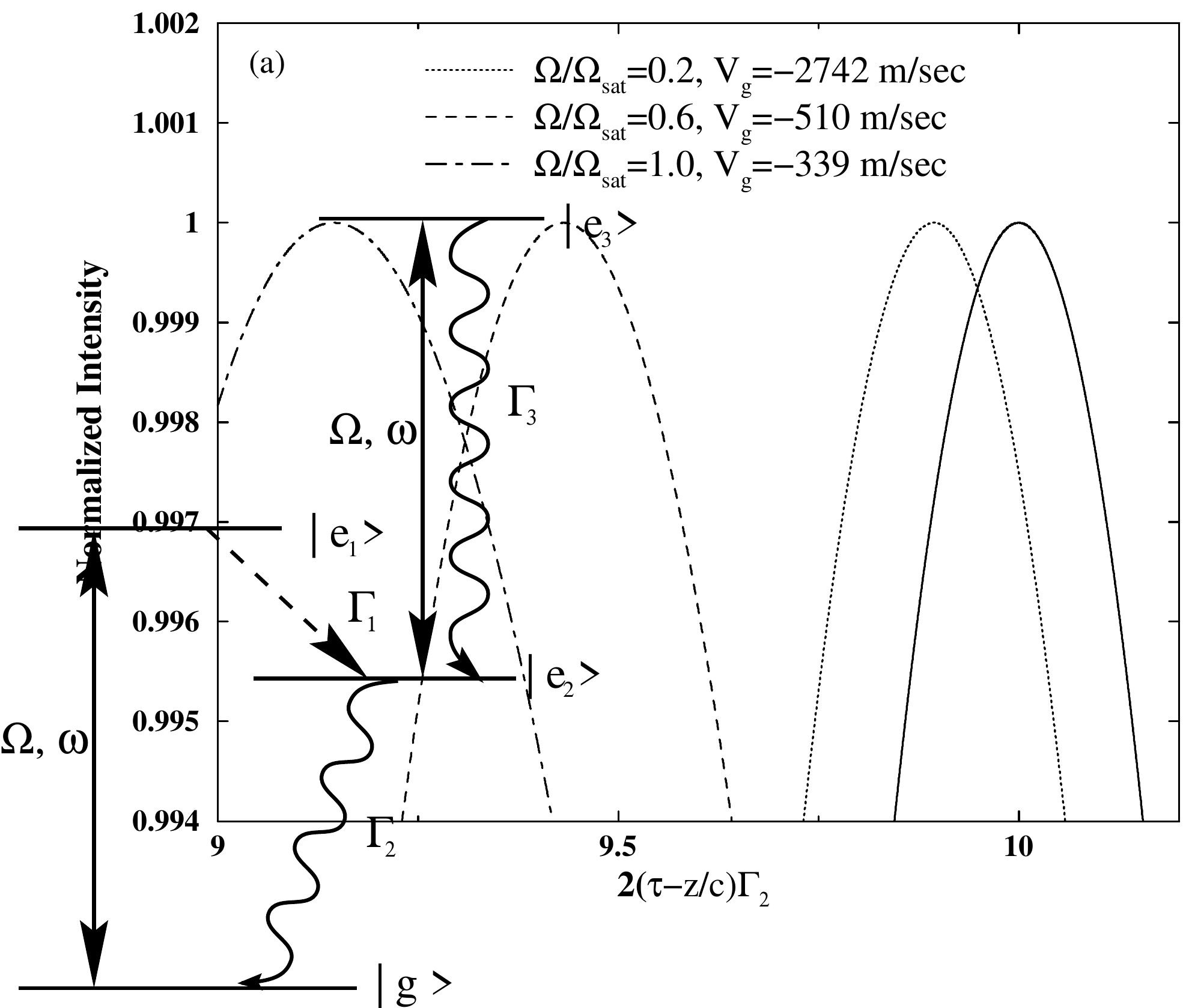




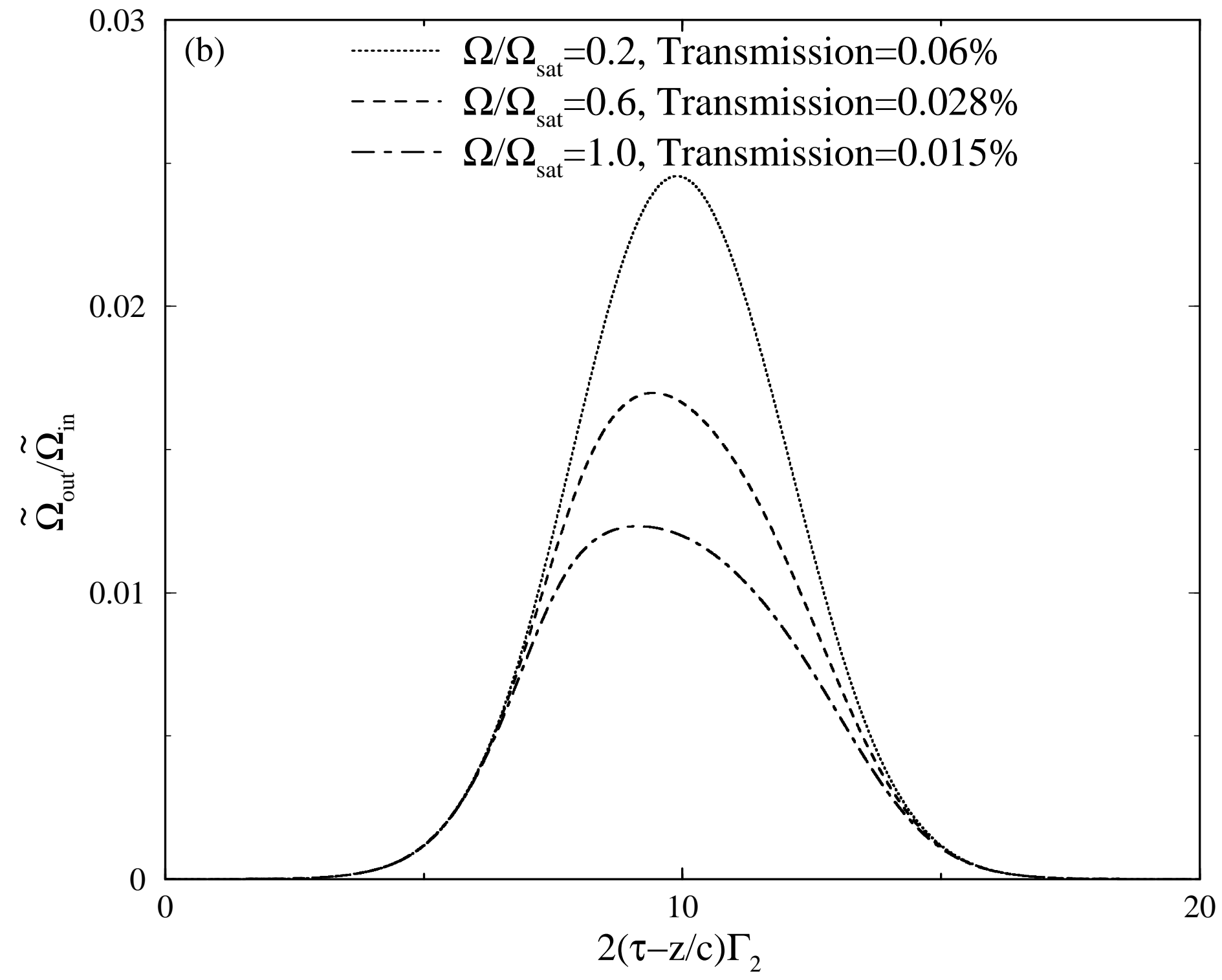

